\title{
Condições clínicas de pacientes com hemofilia assistidos em um hemocentro regional: um estudo transversal
}

RESUMO | O objetivo do estudo foi caracterizar o perfil sociodemográfico e clínico de pacientes com hemofilia acompanhados em um Hemocentro Regional. Estudo epidemiológico de delineamento transversal com coleta de dados por análise documental com 30 prontuários de um Hemocentro Regional da região Centro-Sul do Ceará. Realizou-se análise estatística através do software Microsoft Office Excel囚 2016. O trabalho foi aprovado pelo Comitê de Ética de Pesquisa. Predominou o sexo masculino (27), com idade de 20 a 59 anos, estado civil solteiro, ensino fundamental incompleto, residência em zona rural e renda inferior a 01 salário mínimo. Quanto ao perfil clínico, a hemofilia A na forma grave, apresentam sedentarismo, com histórico de procedimentos transfusionais. A maioria com duas ou mais manifestações clínicas associadas. Com a prevalência apontada entre os participantes, evidencia-se a necessidade de uma assistência integral e equânime, com ênfase nas ações de enfermagem.

Palavras-chaves: Hemofilia; Hemorragia; Epidemiologia.

ABSTRACT | The aim of the study was to characterize the sociodemographic and clinical profile of patients with hemophilia followed at a Regional Blood Center. Epidemiological cross-sectional study with data collection by document analysis with 30 records from a Regional Blood Center in the Center-South region of Ceará. Statistical analysis was performed using Microsoft Office Excel® 2016 software. The work was approved by the Research Ethics Committee. There was a predominance of males (27), aged 20 to 59 years, single marital status, incomplete elementary education, residing in rural areas and income below 01 minimum wage. As for the clinical profile, hemophilia A in severe form, has a sedentary lifestyle, with a history of transfusion procedures. Most have two or more associated clinical manifestations. With the prevalence pointed out among the participants, the need for comprehensive and equitable assistance is evident, with an emphasis on nursing actions.

Keywords: Hemophilia; Bleeding; Epidemiology.

RESUMEN | El objetivo del estudio fue caracterizar el perfil sociodemográfico y clínico de los pacientes con hemofilia seguidos en un Centro Regional de Sangre. Estudio epidemiológico de diseño transversal con recolección de datos por análisis documental con 30 registros de un Centro Regional de Sangre de la región Centro-Sur de Ceará. El análisis estadístico se realizó con el software Microsoft Office Exce|® 2016. El trabajo fue aprobado por el Comité de Ética en Investigación. Predominó el sexo masculino (27), de 20 a 59 años, estado civil soltero, educación primaria incompleta, residente en área rural e ingresos por debajo de 01 salario mínimo. En cuanto al perfil clínico, la hemofilia A en forma severa, tiene un estilo de vida sedentario, con antecedentes de procedimientos transfusionales. La mayoría tiene dos o más manifestaciones clínicas asociadas. Con la prevalencia señalada entre los participantes, se evidencia la necesidad de una atención integral y equitativa, con énfasis en las acciones de enfermería. Palavras claves: Hemofilia; Sangrado; Epidemiología.

\section{Stefane Vieira Nobre}

Enfermeira. Residente em Saúde Coletiva pela Escola de Saúde Pública do Ceará (ESP-CE). ORCID: 0000-0003-4354-2494

\section{José Adelmo da Silva Filho}

Enfermeiro. Mestrando em Enfermagem pelo Programa de Pós-Graduação em Enfermagem da Universidade Regional do Cariri (URCA).

ORCID: 0000-0002-0261-2014

\section{Natália Bastos Ferreira Tavares}

Enfermeira. Diretora da Universidade Regional do Cariri/ Iguatu. Iguatu (CE), Brasil. Mestre em Ciências da Saúde pela Faculdade de Medicina do ABC. São Paulo (SP).

ORCID: 0000-0002-1139-600X

\section{Thiáskara Ramile Caldas Leite}

Enfermeira. Enfermeira assistencial do Hospital Regional do Sertão Central. Quixeramobim (CE), Brasil. Mestre em Enfermagem pelo Programa de PósGraduação em Enfermagem PMAE/URCA. ORCID: 0000-0002-6592-0233

\section{Raimundo Monteiro da Silva Neto}

Bolsista do Programa Institucional de Bolsas de Iniciação Científica PIBIC-CNPq, discente do curso de enfermagem do Centro Universitário de Juazeiro do Norte (UniJuazeiro).

ORCID: 0000-0001-9949-1740

\section{Cicero Rafael Lopes da Silva}

Enfermeiro. Especialista em Enfermagem Dermatológica pelas Faculdades Integradas de Patos (FIP). Professor do Departamento de Enfermagem do Centro Universitário de Juazeiro do Norte (UniJuazeiro).

OIRCD: 0000-0001-8819-5380

\section{Antonio Germane Alves Pinto}

Enfermeiro. Doutor em Saúde Coletiva pela Universidade Estadual do Ceará. Professor Adjunto K do Departamento de Enfermagem da Universidade Regional do Cariri.

OIRCD: 0000-0002-4897-1178

Recebido em: 19/05/2020

Aprovado em: 27/08/2020
INTRODUÇÃO

$\Lambda$ hemofilia é uma patologia hereditária de coagulação sanguínea, decorre do defeito de fatores de coagulação (FC), causa incapacidade de conter um sangramento. É considerada uma doença crônica com consequências físicas e psicológicas, até mesmo incapacitantes, conforme a gravidade da lesão e do nível de deficiência do $\mathrm{FC}^{1}$.

Os sintomas mais comuns da hemofilia são as hemorragias. Estas podem ser classificadas como leves, moderadas e graves e ocorrem principalmente nas articulações, músculos, boca e nariz. Relacionam-se à mortalidade quando acometem o Sistema Nervoso Central, o sistema gastrointestinal, a garganta ou quando são secundárias a traumas graves ${ }^{2}$. 
A disposição geográfica a nível mundial da hemofilia corresponde a aproximadamente um caso para cada 7.500 pessoas com hemofilia tipo A ( $\mathrm{HA})$, e um caso para cada 35 mil pessoas com hemofilia tipo $B(\mathrm{HB})$, sendo ambas proporções voltadas para nascimentos de indivíduos do sexo masculino. No Brasil, a HA é estimada em cerca de $80 \%$ dos casos, sendo mais frequente que a $\mathrm{HB}$. De acordo com o Perfil de Coagulopatias Hereditárias de 2016, o Ceará possuía $4,82 \%$ (488) casos de HA e 2,56\% (51) casos de $\mathrm{HB}^{3}$.

Constitui o tipo de coagulopatia em maior prevalência, configurando-se como um problema importante de saúde pública, pois além de demandar um alto custo por conta do tratamento e profilaxia, também afeta o hemofílico de maneira global. A doença compromete não somente seus aspectos físicos, mas também sociais e psicológicos em virtude de suas complicações a longo prazo, afetando a família e os demais sujeitos envolvidos em seu cotidiano. Tal condição pode ser melhor assistida conhecendo o perfil dos pacientes para melhor direcionar estratégias de assistência à saúde 4 .

Estudos que caracterizam o perfil sociodemográfico de pessoas com hemofilia são necessários pois visam conhecê-los em seu processo de adoecimento. De tal modo é possível estabelecer um plano de cuidado integral com as devidas orientações e tratamentos adequados, amenizando a carga que a doença exerce sobre o hemofílico e sua família?.

Dessa forma, o objetivo do estudo caracterizar o perfil sociodemográfico e clínico de pacientes com hemofilia acompanhados em um Hemocentro com abrangência regional de atendimento.

\section{MÉTODO}

Trata-se de um estudo epidemiológico de delineamento transversal, sobre base de dados primários. O local de realização do estudo foi no departamento de coagulopatias do Hemocentro Regional de
Iguatu, localizado na Região Centro Sul do Estado do Ceará. A escolha do campo de pesquisa justifica-se pelo fato da instituição acima mencionada ser referência no serviço de Hematologia e Hemoterapia na região, respondendo pela política de sangue e pelo atendimento médico das doenças hematológicas em 28 municípios, abrangendo a $17^{\mathrm{a}}$ e $18^{\mathrm{a}}$ Regionais de Saúde e, pois, prestando assistência a uma população de aproximadamente 643.686 habitantes, o que sugere uma ampliação do campo de pesquisa ${ }^{5}$.

O hemocentro regional possui 30 prontuários de pacientes cadastrados, configurando assim a população do estudo. A amostra da pesquisa é do tipo censitária, ou seja, constituída pelos 30 prontuários de pacientes com diagnóstico de hemofilia. O diagnóstico foi confirmado através de exame laboratorial específico para essa condição genética. A amostragem ocorreu mediante aplicação dos critérios de inclusão: prontuários de hemofílicos cadastrados no Hemocentro regional de Iguatu-CE e prontuários de pacientes em acompanhamento clínico, tendo realizado no mínimo uma consulta média no Hemocentro Regional de Iguatu-CE. Foram excluídos da amostra os prontuários de pacientes que possuíam outras coagulopatias e de pacientes que foram a óbito.

As informações epidemiológicas foram levantadas mediante utilização de técnica de análise documental, através dos prontuários de pacientes hemofílicos, obtendo os seguintes dados: sociodemográficos e clínicos.

A coleta de dados ocorreu através da busca nos dossiês dos pacientes hemofílicos cadastrados em novembro de 2016, os dossiês possuem nomes diferentes no Hemocentro. A população em estudo agrupa documentos com informações sobre dados sociodemográficos e triagem clínica. Para a coleta, utilizou-se um formulário com perguntas dicotômicas e de múltipla escolha referentes ao perfil sociodemográfico e clínico de cada paciente. 
TABELA 1- Perfil sociodemográfico de paciente hemofilicos atendidos no Hemocentro de Iguatu-CE em 2016

\begin{tabular}{lcc} 
Variável & N & $\%$ \\
Sexo & & \\
\hline Feminino & 3 & 10 \\
\hline Masculino & 27 & 90 \\
\hline Idade & & \\
\hline $02-19$ & 12 & 40 \\
\hline $20-59$ & 14 & 47 \\
$60-77$ & 4 & 13 \\
\hline
\end{tabular}

\section{Estado civil}

\begin{tabular}{lcc|}
\hline Solteiro & 16 & 53,3 \\
\hline Casado & 14 & 46,6 \\
\hline Escoralidade & 9 & 30 \\
\hline Analfabeto & 1 & 3 \\
\hline Infantil & 2 & 7 \\
\hline Fundamental Completo & 13 & 43 \\
\hline Fundamental Incompleto & 2 & 7 \\
\hline Médio Completo & 3 & 10 \\
\hline Médio Incompleto & & \\
\hline Moradia & 10 & 33 \\
\hline Zona urbana & 20 & 67 \\
\hline Zona rural & & \\
\hline Renda familiar & 14 & 47 \\
\hline Menos de um salário & 13 & 43 \\
\hline Salário mínimo & 3 & 10 \\
\hline Mais de um salário & 30 & $100 \%$ \\
\hline Total & &
\end{tabular}

Fonte: Iguatu-CE, 2020. HEMOCE/IGUATU-CE, 2016

TABELA 2- Condições clínicas e funcionais dos pacientes atendidos do Hemocentro Regional de Iguatu-CE em 2016, Iguatu-CE, 2020.

\section{Variável}

Tipo de hemofilia

Tipo A

Tipo B

\section{Gravidade da hemofilia}

$\begin{array}{lcl}\text { Leve } & 7 & 23 \\ \text { Moderada } & 7 & 23 \\ \text { Grave } & 16 & 53\end{array}$

\section{Faz parte do Programa de Dose Domiciliar}

$$
\text { Sim }
$$

Não

N $\%$

22

73

$\begin{array}{ll}22 & 27\end{array}$

$27 \quad 90$

10

As variáveis inclusas no estudo foram: ambos sexos, estado civil (todos), faixa etária (2-77 anos), residentes em zonas urbanas e rurais, escolaridade até o ensino médio, todos os tipos de gravidade da hemofilia e que já receberam transfusão sanguínea.

$\mathrm{Na}$ fase da análise foi recorrido à estatística descritiva por meio do Excel 2016. Os dados foram apresentados através de tabelas com frequências absoluta e relativa.

Os procedimentos e condutas adotados no presente estudo seguiram as recomendações éticas e legais da Resolução $n^{\circ} 466$, de 12 de dezembro de 2012. O estudo foi aprovado pelo Comitê de Ética e Pesquisa da Universidade Regional do Cariri - URCA, obtendo o parecer de número: 1.827 .856 e CAAE: 58595116.0 .0000 .5055 .

\section{RESULTADOS}

Considerando o número de prontuários cadastrados de pacientes com hemofilia no lócus estudado, obteve-se em predominância: homens 27 (90\%), adultos de 20 a 59 anos (47\%), solteiros (54,3\%), que cursaram o fundamental incompleto (43\%), residem em zona rural (67\%) e sobrevivem com uma renda familiar inferior a um salário mínimo (47\%).

A tabela 2 apresenta as características clínicas e funcionais dos pacientes. O hemofílico atendido no hemocentro de Iguatu-CE possui hemofilia A (73\%), sendo de magnitude grave $(53 \%)$, recebe dose domiciliar (90\%), já foi transfundido $(70 \%)$, apresenta duas ou mais manifestações clínicas (83\%), possui complicações osteoarticulares (67\%) e é sedentário $(83 \%)$

\section{DISCUSSÃO}

A maioria dos pacientes hemofílicos do estudo eram do sexo masculino 27 (90\%). Este achado é explicado pela própria fisiopatologia da doença ao tra- 


\section{Já foi submetido a transfusões clínicas}

\begin{tabular}{|lcc|}
\hline Sim & 21 & 70 \\
\hline Não & 9 & 30 \\
\hline Ocorrêcia das manifestações clínicas & & \\
\hline Nenhuma & 3 & 10 \\
\hline 1 manisfestação & 2 & 7 \\
\hline 2 ou mais manifestações & 25 & 83 \\
\hline Presença de Complicações Osteoarticulares & & \\
\hline Sim & 20 & 67 \\
\hline Não & 10 & 33 \\
\hline Prática de Atividade Física & & \\
\hline Sim & 5 & 17 \\
\hline Não & 25 & 83 \\
\hline
\end{tabular}

Fonte: HEMOCE/IGUATU, 2016.

zer que as hemofilias são transmitidas de mãe para filho, como herança genética ${ }^{6,7}$. A faixa etária prevalente no estudo compreendeu o intervalo de 20-59 anos $(47 \%)$, corroborando com estudos que trazem que a população com esta faixa etária tende a ser a mais acometida pela doença $a^{7,9}$.

A pesquisa apontou que a maioria dos hemofílicos eram solteiros, pode-se inferir que este achado decorre da faixa etária ainda jovem dos pacientes, de modo que muitos acabam passando um maior tempo com os pais mediante a dependência em relação aos seus cuidados ${ }^{8}$.

Revelou-se uma baixa escolaridade, tendo em vista que recebem o diagnóstico precocemente e até mesmo antes de começarem a frequentar a escola. Em virtude do caráter severo da doença os hemofílicos tendem a isolar-se do convívio social por estarem limitados a praticar atividades vigorosas e brincadeiras. Entretanto a doença não ocasiona nenhuma deficiência intelectual, tornando-se indispensável que não só a equipe de saúde, mas também a família o estimule ao convívio com os demais indivíduos, principalmente em âmbito escolar ${ }^{9}$.

Em relação à procedência geográfica dos pacientes acompanhados, a maioria está localizada em zona rural, ocasionando dificuldade no processo de locomoção até a cidade para as consultas e a realização do tratamento. Um estudo realizado em Pelotas obteve que $41,7 \%$ residia no município onde encontrava-se o hemocentro regional, os demais $58,3 \%$ residiam em outros municípios?

Os dados relativos à renda encontravam-se em desatualização de acordo com o valor de salário mínimo que hoje é fornecido, R\$ 1045,0010. Registros notificam que havia o recebimento de benefício para àqueles com comprometimento físico, porém não continha o valor recebido.A HA ocorreu em maior frequência no estudo, estando de acordo com outros estudos que trazem que esta corresponde a $80-85 \%$ dos casos no Brasil ${ }^{3,7,11}$.

No perfil traçado por esse estudo, a gravidade da hemofilia, mostrou-se grave, corroborando com estudos realizados em outras localidades, como no Hemocentro Regional de Pelotas-HEMOPEL ${ }^{7}$. Em uma pesquisa realizada na Colômbia obteve-se que em uma amostra de 33 pacientes hemofílicos, a severidade da doença no nível grave representou $82 \%{ }^{12}$.

O método principal e mais seguro utilizado atualmente para o tratamen- to consiste na administração de FC VIII e IX sendo adequados aos tipos A e B respectivamente. Estes funcionam como uma forma de suprir a falta dos FC não produzidos pelo organismo do hemofílico ${ }^{13-14}$. Desde 1999, o Ministério da Saúde através do Programa de Dose Domiciliar objetiva tornar o hemofílico mais autônomo e participativo quanto a sua terapêutica. Também disponibiliza uma capacitação aos pacientes ou cuidadores para a infusão do FC em domicílio através de venóclises ${ }^{13-14}$. O estudo trouxe que $90 \%$ dos hemofílicos participam do programa.

Outro método também utilizado como forma de tratamento consiste na transfusão de hemoderivados. Os riscos envolvidos consistem em efeitos adversos ou reações transfusionais, que podem ocorrer durante ou após a transfusão. Configuram-se mais raras as reações transfusionais graves, sendo as leves, os mais comuns e presentes durante a rotina de atuação da enfermagem 15,16,17.

Os prontuários analisados trouxeram como manifestações clínicas: hemartroses, hematúria, melena, hematêmese, menorragia, sangramentos após traumas e procedimentos odontológicos, sangramento pós-parto, sangramento oral e cutâneo e epistaxe. Em estágios graves, os episódios de hemorragias espontâneas predominantemente em músculos e articulações. Entretanto não costumam sangrar, havendo casos de pacientes que nunca relatam problemas com sangramentos ${ }^{18}$.

Referente as complicações osteoarticulares, essas incluem atrofias e proliferação dos ossos e irregularidades da superfície articular com formação osteófita, desenvolvimento acelerado e supercrescimento da epífise causadas pelo excessivo fluxo sanguíneo, necrose óssea e formação cística, como também impedimento do crescimento do osso resultante da interferência da nutrição óssea. As articulações frequentemente são acometidas são as do tornozelo, 
joelho, quadris, ombro, cotovelo e punho. A estas complicações afetam as articulações que são utilizadas no desempenho das atividades básicas, corre o risco de sequelas irreversíveis no aparelho osteoarticular perda da movimentação do membro ${ }^{19,20}$.

Atividades que envolvam correr e pular podem desencadear várias complicações osteoarticulares, podendo levar a graves sequelas. Desta forma, resta ao hemofílico a adesão ao tratamento permanente e obter melhora em sua qualidade de vida. Os pacientes da pesquisa são sedentários, reforçando o problema dos hemofílicos quanto ao comprometimento de estruturas de sus- tentação e movimentação constantes, como as articulações ${ }^{20-21}$.

Frente a esta problemática, é importante ofertar orientações quanto a prática regular e adequada de exercícios, pois estes serão benéficos para o funcionamento adequado do sistema osteolocomotor, evitando que o paciente se torne sedentário e consequentemente venha a ter complicações futuras ${ }^{20-21}$

\section{CONCLUSÃO}

Os hemofílicos necessitam de uma assistência integral e diferenciada mediante suas particularidades. Para isso, é necessária a produção de estudos que busquem conhecer o perfil do hemofílico e de sua doença, para subsidiar a terapêutica por parte dos profissionais. Dentre estes, destaca-se o enfermeiro com sua capacidade de planejar ações de cuidado em saúde atendendo a individualidade do sujeito e envolvendo a família, a equipe e os demais segmentos sociais no conhecimento sobre a doença e as formas de lidar com ela.

\section{AGRADECIMENTOS}

Agradecemos ao Fundo Estadual de Combate à Pobreza do estado do Ceará, pelo financiamento deste estudo.

\section{Referências}

1. Booth J, Oladapo A, Walsh S, O'Hara J, Carroll L, Diego DAG et al. Real-world comparative analysis of bleeding complications and health-related quality of life in patients with haemophilia A and haemophilia B. Haemophilia. 2018; 24: e322-e327. DOI: https://doi.org/10.1111/hae.13596

2.World Federation of Hemophilia [homepage na internet]. Montreal: World Federation of Hemofilia. [Atualizada em 2012 Mai; acesso em 2020 Abr 03]. Sintomas e diagnóstico. Disponivel em: http://www.wfh.org/en/page.aspx?pid=640. 3.Ministério da Saúde; Secretaria de Atenção à Saúde; Departamento de Atenção Especializada e Temática. Perfil das coagulopatias hereditárias no Brasil: 2016. Brasília: Ministério da Saúde, 2018.

4.Lavaut-Sánchez, K. Importancia del diagnóstico genético en hemofilia. Revista Cubana de Hematología, Inmunología y Hemoterapia. 2017; 33 (S1). Disponível em: http://revhematologia.sld.cu/index.php/hih/article/viewFile/810/622

5. Centro de Hematologia e Hemoterapia do Ceará [homepage na internet]. Ceará: Centro de Hematologia e Hemoterapia do Ceará. [Acesso em 2020 Abr 04]. Hemocentro Regional de Iguatu. Disponivel em: http://www.hemoce.ce.gov.br/ index.php/hemocentro-regional-iguatu.

6.Sousa JG, Vieira RTF, Duarte ARP, Figueira MCS, Jacob LMS, De Melo MC. Qualidade de vida dos portadores de hemofilia a em um hemocentro na região amazônica. Revista Intellectus, 2018; 1(44): 33-45. ISSN 1679-8902.

7.Feijó AM, Schwartz E, Lise F, Santos BP, Spagnolo LML. Características sociodemográficas de homens com hemofilia no sul do Brasil. Cienc Cuid Saude, 2018, 17(4):2-8. DOI: 10.4025/cienccuidsaude.v17i4.45048.

8.Apolinário LA, Rodrigues LR. Mães de crianças e adolescentes hemofilicos e suas concepções sobre maternidade. REAS [Internet], 2013; 2(3):42-52. ISSN: 2317-1154.

9.Martínez-Sanchez LM, Jonathan CV, Sergio RP, Rodriguez-Gázquez MLO. Perfil clínico y epidemiológico de pacientes con hemofilia registrados en la Liga de Hemofílicos de Antioquia (Colombia). Rev Méd Risaralda. 2017; 23 (1):34-37. ISSN 0122-0667.

10.Departamento Intersindical de Estatística e Estudos Socioeconômicos [homepage na internet]. São Paulo: Departamento Intersindical de Estatística e Estudos Socioeconômicos. [Acesso em 2020 Abr 03]. Nota Técnica 218 Salário Mínimo. Disponivel em:https://www.dieese.org.br/notatecnica/2019/notaTec218SalarioMinimo.html.

11.Souza VN, Pereira AS, Vesco NL, Brasil BMBL, Barbosa SM, Viana CDMR. Nurses' knowledge of hemophilia clinics of on systematization nursing care. $J$ Nurs UFPE on line. 2016; 10(5):1654-1662. DOI: 10.5205/reuol.9003-78704-1-

\section{SM.1005201611.}

12.Yunis LK, Linares A, Cabrera E, Yunis JJ. Systematic molecular analysis of hemophilia A patients from Colombia. Genet Mol Biol Ribeirão Preto. 2018; 41(4):750-757. DOl: http://dx.doi.org/10.1590/1678-4685-gmb-2017-0072. 13.Ministério da Saúde; Secretaria de Atenção à Saúde; Departamento de Atenção Especializada. Manual de Dose Domiciliar para Tratamento das Coagulopatias Hereditárias. Brasília: Ministério da Saúde; 2007.

14.Ferreira D, Silva ELP, Borges DDL, Santos GM, Noueira TA, Silva HJN, Oliveira $K B V$. Prevalência das coagulopatias hereditárias nos portadores atendidos no centro de hematologia e hemoterapia do Piauí - HEMOPI. Braz J Surg Clin Res. 2018; 24(1):56- 60. ISSN: 2317-4404.

15. Silva EM, Vieira CA, Silva FO, Ferreira EV. Desafios da enfermagem diante das reações transfusionais. Rev enferm UERJ. 2017; 25:e11552. DOI: http://dx.doi. org/10.12957/reueri.2017.11552.

16.Forster F, Câmara AL, Moraes CLK, Honório MT, Mattia D, Lazzari DD. Percepção dos enfermeiros quanto à assistência de enfermagem no processo transfusional. Enferm Foco. 2018; 9(3): 71-75. DOI: https://doi.org/10.21675/ 2357-707X.2018.v9.n3.1509.

17. Cherem EO, Alves VH, Rodrigues DP, Souza FDL, Guerra JVV, Maciel VL. Saberes do enfermeiro para o cuidado no processo transfusional em recém-nascidos. Rev Gaúcha Enferm. 2017; 38(1): e63557. DOI: http://dx.doi.org/10.1590/19831447.2017.01.63557.

18.Santos RS, Figueirôa GR, Machado BA, Mamede AGS, Gois LML, Silva Junior MCM. Frequência de alterações posturais em hemofilicos. Rev Pesq Fisio Salvador, 2018;8(1):24-36.DOI:10.17267/2238-2704rpf.v8i1.1616.

19.Forsyth AL, Witkop M, Lambing A, Garrido C, Dunn S, Cooper DL, Nugent DJ. Associations of quality of life, pain, and self-reported arthritis with age, employment, bleed rate, and utilization of hemophilia treatment center and health care provider services: results in adults with hemophilia in the HERO study. Patient Prefer Adherence. 2015; 29(9):1549-1560. DOI: 10.2147/PPA.S87659. PMID: 26604708; PMCID: PMC4631419.

20.Goto M, Takedani H, Yokota K, Haga N. Strategies to encourage physical activity in patients with hemophilia to improve quality of life. Journal of blood medicine. 2016; 7: 85-98. DOI: 10.2147/JBM.S84848.

21.Targino Júnior MA, Leite Filho MAA, Montenegro RC, Barbosa EL. Antropometria e força muscular de indivíduos hemofilicos da cidade de João Pessoa-PB. RBPFEX [Internet]. 2017; 11(69):743-7. Disponível em: http://www.rbpfex.com. br/index.php/rbpfex/article/view/1261. 